\title{
THE OPTIMAL STRUCTURAL DESIGN OF THE HUMAN SPINAL INTERVERTEBRAL DISC
}

\author{
D.N. GHISTA ${ }^{1}$, S.C. FAN ${ }^{2}$, I. SRIDHAR ${ }^{3} \&$ K. RAMAKRISHNA ${ }^{3}$ \\ ${ }^{1}$ University of New South Wales Asia, Singapore. \\ ${ }^{2}$ School of Civil and Environmental Engineering, Nanyang Technological University, Singapore. \\ ${ }^{3}$ School of Mechanical and Aerospace Engineering, Nanyang Technological University, Singapore.
}

\begin{abstract}
The intervertebral disc (IVD) acts as a shock-absorbing unit and effectively contains its lateral and axial deformations while providing the necessary flexibility to the spine. These attributes are due to the stress-stiffening material (elastic modulus) property of the annulus, caused by the pressure developed in the nucleus pulposus (NP). Hence, one of the biomechanical roles of the NP in the IVD is to stress the annulus while the IVD is loaded. In this paper, a closed-form solution of the IVD (with NP, i.e. a healthy IVD, and without NP, i.e. a nucleotomized IVD) under compressive loading is developed. Based on the analysis, it is observed that the deformations of the IVD do not increase in proportion to the load. Rather, the rate of increase in deformation decreases as the load increases. This is a key optimal feature because it means that deformations are contained and therefore stability is maintained. Further, it is shown that the nucleotomized IVD deforms more than the healthy IVD. This means that the nucleotomized IVD will have higher chances of collapse than the healthy IVD for the same level of loading. This result is a contra-indication for nucleotomy. Our proposal is to place a biocompatible gel-filled balloon to simulate the beneficial effects of the NP.

Keywords: deformation, internal pressure, intervertebral disc, nucleotomy, nucleus pulposus, stress analysis, stress stiffening solid, thick-walled cylinder, uniaxial compression.
\end{abstract}

\section{CONCEPT OF THE INTERVERTEBRAL DISC AS AN OPTIMAL STRUCTURE}

The human spine is made up of alternating vertebral body (VB) and intervertebral disc (IVD). In our earlier paper [1], we have shown how the VB is designed as an optimal lightweight structure because of its hyperboloid shape. An additional feature of the spine as a structure is its ' $S$ ' shaped configuration. This configuration has evolved to enable a human being to stand and move in an upright configuration while supporting the internal organs and providing rigidity for the functional role of sitting and squatting. However, the ' $\mathrm{S}$ ' shaped configuration also acts as a shock absorber. In this function of the spine to act as a flexible shock-absorbing and protective structure, the IVD has an important role.

The IVD, as the principal component of the intervertebral joint (shown in Fig. 1a), sustains and transmits compressional, bending and torsional loadings. It is centrally pressurized by the nucleus pulposus (NP) and surrounded by the annulus (Fig. 1b). The annulus fibres are oriented helically, at almost $30^{\circ}-50^{\circ}[2,3]$. Even under torsion, the torsional shear stresses on a disc element will result in diagonally oriented tensile and compressive stresses. It is revealing that these tensile stresses due to torsion of the disc can thus be directly absorbed by these angled fibres of the annulus. Thus, the IVD is ideally designed for compression and bending as well as for torsion [4-12].

The IVD functions as the shock-absorbing component of the spinal unit, comprising two adjacent VBs on either side of the IVD. Additionally, the central portion of the VB end-plate functions as a diaphragm, through which (under compressive loading) NP fluid moves out of the disc into the VB, thereby helping to draw nutrition (as shown in Fig. 2a and b) into the disc upon removal of the loading. However, under rapidly applied loading, the VB end-plate offers resistance to the intrusion of fluid into the VB blood compartment, thereby lending a shock-absorbing property to the disc. Indeed, the IVD can be regarded as an effective viscoelastic shock-absorbing structure [13].

From a biomechanical viewpoint, the NP has another very important role, namely to contain the disc axial and radial deformations. The causative mechanism is that when the disc is loaded in axial 


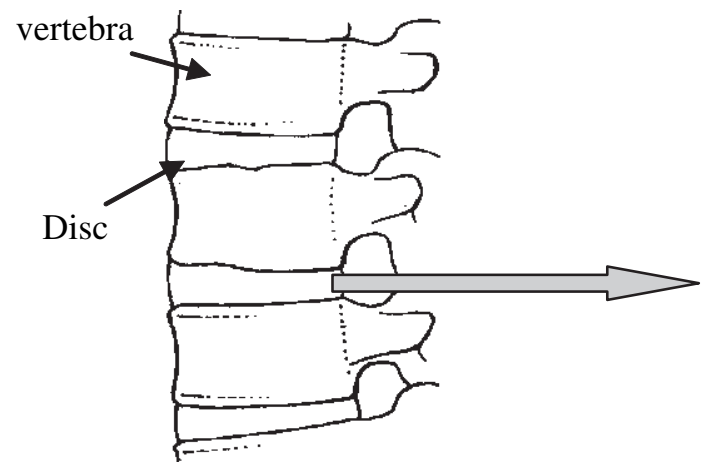

(a)

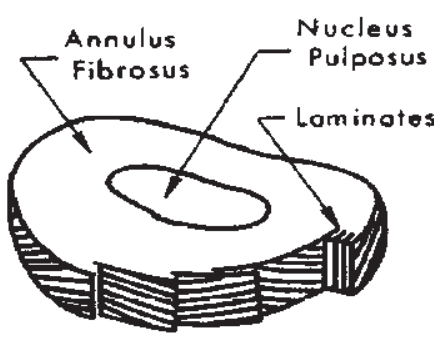

(b)

Figure 1: (a) The location of the IVD within the spinal column. (b) Schematic representation of the disc structure. The NP is surrounded by annulus fibrosus. This outer layer has a lamellar structure with highly ordered collagen fibres [4].

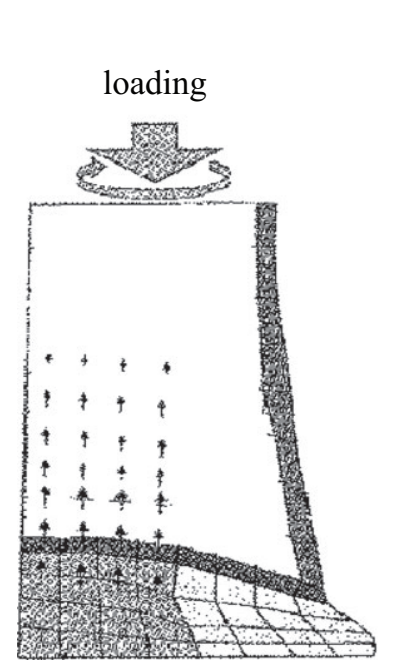

(a)

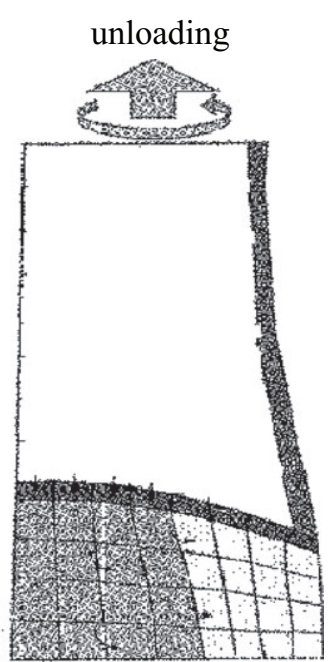

(b)

Figure 2: Nutrition mechanism of the disc (a) during loading, the NP enters the cancellous VB, and (b) during unloading, the NP draws nutrition into the disc [13].

compression (or bending or torsion), the NP fluid gets pressurized and stresses the surrounding annulus. The annulus is a stress-stiffening solid, such that its elastic modulus $(E)$ increases with the increase in stress (its stress-strain property is shown in Fig. 3b) [14, 15]. Hence, under increased loadings, its elastic modulus value also increases, so that the deformations are thereby contained.

The IVD's stress-deformation characteristics have been effectively analysed by finite element analysis [14]. Herein, an elasticity model of the disc as a closed thick-walled fluid-filled cylinder is employed to determine its stress and deformations under uniaxial compressive loading and demonstrates the role of the NP in containing the disc deformations. It is also demonstrated that 
the nucleotomized disc will undergo larger deformations than the normal disc, for the same levels of loading, thereby drawing attention to the efficacy of nucleotomy to treat a ruptured disc and associated back pain.

The stress-dependent Young's modulus of the disc annulus can be represented as [14]

$$
E=E_{0}+375.3 \sigma^{0.473}
$$

where $E_{0}$ (the residual Young's modulus) $=4.2 \mathrm{MPa}$ and the stress $\sigma$ is expressed in MPa. This constitutive equation is employed to determine the $E$ value for the uniaxially compressed disc. This constitution of the spinal disc, wherein the stress-dependent Young's modulus of its annulus encloses the NP, gives it a key self-reinforcing design property. The closest man-made self-reinforcing structure is a car tyre, which makes it lighter as well as lends it a shock-absorbing property.

The disc annulus is assumed to be isotropic, so that $E_{z}=E_{r}=E_{\theta}=E$. As the disc gets compressed (by increasing the applied compressive force $F$ ), the annulus stresses $\left(\sigma_{z}, \sigma_{r}\right.$ and $\left.\sigma_{\theta}\right)$ keep increasing. For each updated value of $E$ for the enhanced stress state (in response to increasing values of the compression force $F$ on the disc), $\sigma$ (in eqn (1D) is taken to be equal to the maximum value of the principal stress (which happens to be the axial stress $\sigma_{z}$ ). For this relationship, as the disc is loaded, the annulus stress state $\sigma=\left(\sigma_{z}\right)$ increases. Correspondingly, its $E$ increases, to thereby contain the disc deformations.

In this paper, the mechanism of disc deformation containment for vertical loading is delineated. Compressive loading $(F)$ on the disc causes compressive axial stress $\left(\sigma_{z}\right)$ in the annulus and also pressurizes NP fluid, which then exerts hydrostatic pressure $\left(p_{i}\right)$ and hence compressive radial stress $\sigma_{r}$ on the annulus. This radial pressure or stress $\left(\sigma_{r}\right)$ in turn causes circumferential tensile stress $\left(\sigma_{\theta}\right)$ in the annulus. These stresses in turn influence the strain state in the disc through the elastic modulus and, hence, the axial and radial deformations of the disc by virtue of eqn (1). Hence, the effect of the NP hydrostatic pressure is to stress the disc annulus and enhance the value of its $E$. This in turn stiffens the disc (according to eqn (1) and enables it to bear heavy loads without large axial and lateral deformations.

\section{ANALYSIS: DISC STRESSES, DISPLACEMENTS AND DEFORMED GEOMETRY}

The disc is considered to be a thick-walled isotropic cylinder whose geometry and deformations are depicted in Fig. 3a. In this analysis, linear elasticity formulations of stress-strain constitutive relations have been employed. Under compressive loading of the order of $2000 \mathrm{~N}$, the deformations are of the order of $1 \mathrm{~mm}$. This result has been obtained by Fagan et al. [6, 16] and is shown in Fig. 3b. Hence, in order to compute the disc deformations under compressive loading, small incremental loadings are adopted so that the resulting strains are infinitesimal. Likewise, for each incremental load state, (1) the NP pressure is determined, (2) the incremental stresses and the total stress state are computed, (3) the disc material modulus value is revised as per eqn (1) and (4) the disc deformations are determined and its geometry is updated.

Stress equilibrium equations: Let $u$ be the radial displacement and $w$ be the axial displacement, as shown in Fig. 3. Because of the axial symmetry of the disc geometry and loading conditions, the shear stresses and the circumferential displacement are identically equal to zero. Thus, the stressequilibrium equations are

$$
\begin{array}{ll}
\text { Radial direction } & \frac{\mathrm{d} \sigma_{r}}{\mathrm{~d} r}+\frac{\sigma_{r}-\sigma_{\theta}}{r}=0 \\
\text { Axial direction } & \frac{\mathrm{d} \sigma_{z}}{\mathrm{~d} z}=0 .
\end{array}
$$




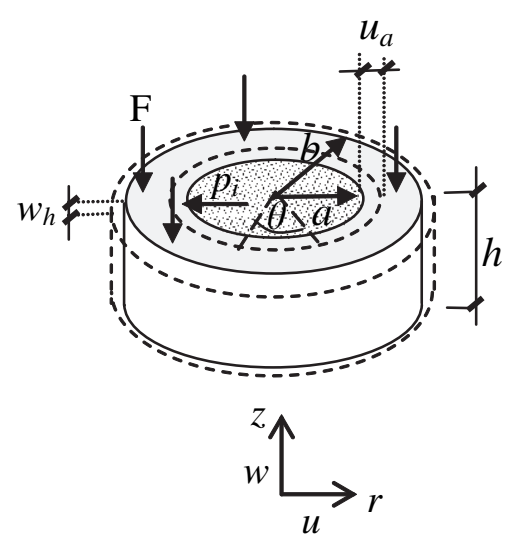

(a)

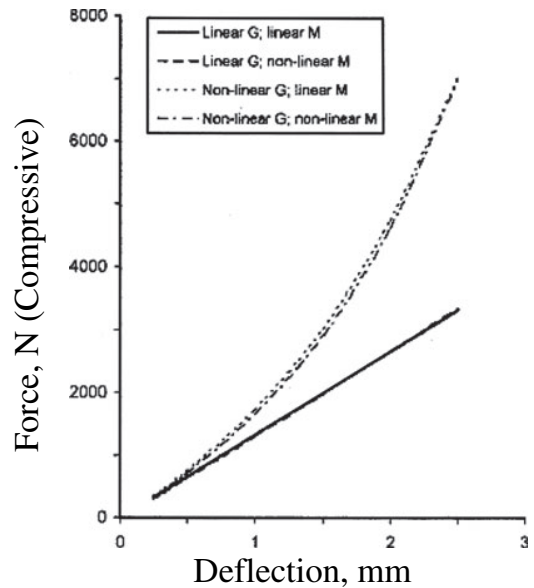

(b)

Figure 3: (a) Geometry and deformation variables of the spinal disc loaded in compressive force $F$. Note that $u$ is depicted as expansive radial deformation while $w$ is depicted as shortening axial deformation. (b) Comparison of the effects of including linear and non-linear material $(\mathrm{M})$ and geometry $(\mathrm{G})$ solution options on the compressive behaviour of the disc [6].

The strain-displacement relations are

$$
\begin{array}{ll}
\text { Radial strain } & \varepsilon_{r}=\frac{\sigma_{r}}{E}-\frac{\nu\left(\sigma_{\theta}+\sigma_{z}\right)}{E}=\frac{\mathrm{d} u}{\mathrm{~d} r}, \\
\text { Circumferential strain } & \varepsilon_{\theta}=\frac{\sigma_{\theta}}{E}-\frac{\nu\left(\sigma_{z}+\sigma_{r}\right)}{E}=\frac{u}{r} \\
\text { Axial strain } & \varepsilon_{z}=\frac{\sigma_{Z}}{E}-\frac{\nu\left(\sigma_{r}+\sigma_{\theta}\right)}{E}=\frac{\mathrm{d} w}{\mathrm{~d} z},
\end{array}
$$

wherein the annulus material modulus $(E)$ is adopted to be isotropic.

The disc material's constitutive stress-strain relations in terms of the disc material's Young's modulus $(E)$ and Poisson's ratio $(v)$ are hence given in the radial direction as

$$
\sigma_{r}=\frac{E}{1+v}\left(\frac{v}{(1-2 v)}\left(\frac{d u}{d r}+\frac{u}{r}+\frac{d w}{d z}\right)+\frac{d u}{d r}\right),
$$

in the circumferential direction as

$$
\sigma_{\theta}=\frac{E}{1+v}\left(\frac{v}{(1-2 v)}\left(\frac{d u}{d r}+\frac{u}{r}+\frac{d w}{d z}\right)+\frac{u}{r}\right)
$$

and in the axial direction as

$$
\sigma_{z}=\frac{E}{1+v}\left(\frac{v}{(1-2 v)}\left(\frac{d u}{d r}+\frac{u}{r}+\frac{d w}{d z}\right)+\frac{d w}{d z}\right) .
$$

Note that $\sigma_{\theta}, \sigma_{r}, \sigma_{z}$ are adopted to be positive for tensile stress. 
Now, by substituting the constitutive relations in eqn $(5 \mathrm{a}-\mathrm{c})$ into the equilibrium equations (2) and (3), two partial differential equations in displacements $u$ and $w$ are obtained, as follows

$$
\begin{gathered}
\frac{\mathrm{d}}{\mathrm{d} r}\left(\frac{v}{1-2 v}\left(\frac{\mathrm{d} u}{\mathrm{~d} r}+\frac{u}{r}+\frac{\mathrm{d} w}{\mathrm{~d} z}\right)+\frac{\mathrm{d} u}{\mathrm{~d} r}\right)+\frac{1}{r}\left(\frac{\mathrm{d} u}{\mathrm{~d} r}-\frac{u}{r}\right)=0 \\
\frac{\mathrm{d}}{\mathrm{d} z}\left(\frac{v}{1-2 v}\left(\frac{\mathrm{d} u}{\mathrm{~d} r}+\frac{u}{r}+\frac{d w}{\mathrm{~d} z}\right)+\frac{\mathrm{d} w}{\mathrm{~d} z}\right)=0
\end{gathered}
$$

The solutions of eqn (6a) and (6b) can be expressed as

$$
\begin{aligned}
& u=\frac{A}{r}+B r, \\
& w=C z+D,
\end{aligned}
$$

where, A, B, C and D are the constants of integrations. These constants can be determined by applying appropriate boundary conditions.

As the NP is incompressible [2], its volume after deformation is unchanged, so that

$$
\pi a^{2} h=\pi\left(a+u_{a}\right)^{2}\left(h-w_{h}\right) .
$$

This can be simplified by neglecting higher-order terms $\left(u_{a} w_{h}\right.$ and $\left.u_{a}^{2} w_{h}\right)$, to yield

$$
2 \pi a h u_{a}-\pi a^{2} w_{h}=0 \quad \text { or } \quad u_{a}=\left(\frac{a}{2 h}\right) w_{h}
$$

It is to be noted that according to deformation as per Fig. $3 \mathrm{a}, \mathrm{w}_{h}$ is the shortening deformation at $z=h$, while $u_{a}$ is the radial expansion deformation at $r=a$.

The appropriate boundary conditions for solving eqns (7) and (8) are

$$
\begin{gathered}
u_{r=a}=u_{a}=\frac{A}{a}+B a, \\
\sigma_{r}=0 \quad \text { at } r=b, \\
w=0 \quad \text { at } z=0, \\
w=-w_{h} \quad \text { at } z=h .
\end{gathered}
$$

Using the boundary conditions from eqn (10) and utilizing eqns (5), (7) and (8), the constants in eqns (7) and (8) are obtained as

$$
\begin{gathered}
A=\frac{(1-2 v) u_{a} a b^{2}}{b^{2}+a^{2}(1-2 v)}, \\
B=u_{a}\left(\frac{a^{2}(1-2 v)+2 v b^{2}}{a b^{2}+a^{3}(1-2 v)}\right), \\
C=-\frac{w_{h}}{h}=-\frac{2 u_{a}}{a}, \\
D=0 .
\end{gathered}
$$


Using the constants $A, B, C$ and $D$, the stresses in eqn (5) can be represented by

$$
\begin{gathered}
\sigma_{r}=\frac{E}{1+v}\left(\frac{v(2 B+C)}{(1-2 v)}+B-\frac{A}{r^{2}}\right) \\
=-\frac{E}{1+v}\left(\frac{u_{a} a(1-2 v)}{a^{2}(1-2 v)+b^{2}}\right)\left(\frac{b^{2}}{r^{2}}-1\right), \\
\sigma_{\theta}=\frac{E}{1+v}\left[\frac{v(2 B+C)}{(1-2 v)}+B+\frac{A}{r^{2}}\right] \\
=\frac{E}{1+v}\left(\frac{u_{a} a(1-2 v)}{a^{2}(1-2 v)+b^{2}}\right)\left(\frac{b^{2}}{r^{2}}+1\right), \\
\sigma_{z}=\frac{E}{(1+v)}\left(\frac{v(2 B+C)}{(1-2 v)}+C\right)=-\frac{2 u_{a} E}{a(1+v)}\left[\frac{a^{2}(1-2 v)+b^{2}(1+v)}{a^{2}(1-2 v)+b^{2}}\right] \\
=-\frac{w_{h} E}{(1+v)}\left[\frac{a^{2}(1-2 v)+b^{2}(1+v)}{a^{2}(1-2 v)+b^{2}}\right] .
\end{gathered}
$$

Then, from eqns (7), (11a) and (11b), the radial displacement is given by

$$
u_{r}=\frac{A}{r}+B r=\frac{u_{a}}{a r}\left(\frac{a^{2} b^{2}(1-2 v)+r^{2}\left[a^{2}(1-2 v)+2 v b^{2}\right]}{a^{2}(1-2 v)+b^{2}}\right)
$$

and hence $u_{b}$ (at $r=b$ ) is given by

$$
u_{b}=\frac{2 b u_{a}}{a}\left(\frac{a^{2}(1-2 v)+v b^{2}}{a^{2}(1-2 v)+b^{2}}\right) .
$$

It is to be noted (from eqn (12c)) that $\sigma_{z}$ is uniform throughout the disc and the minus sign implies that $\sigma_{z}$ is compressive.

\section{STRESS ANALYSIS OF THE HEALTHY DISC UNDER COMPRESSION}

For an axially applied force $F$ (as illustrated in Fig. 4), the equilibrium equation is

$$
F=\pi a^{2} \sigma_{f}-\pi\left(b^{2}-a^{2}\right) \sigma_{z}
$$

where $\sigma_{f}$ is the hydrostatic pressure in the fluid and $\sigma_{z}$ is the axial stress in the annulus (as shown in Fig. 4). Its sign is taken to be negative in eqn (14), because positive $\sigma_{z}$ is considered as tensile.

Because the disc height $(h)$ is small, $\sigma_{f}$ is approximately constant, and hence:

$$
\sigma_{f}=-\left.\sigma_{r}\right|_{r=a}=p_{i} \quad(\text { the pressure in the NP). }
$$

Based on eqns (15) and (12a),

$$
p_{i}=\frac{E(1-2 v)}{(1+v)}\left(\frac{u_{a}}{a}\right)\left(\frac{b^{2}-a^{2}}{a^{2}(1-2 v)+b^{2}}\right) .
$$




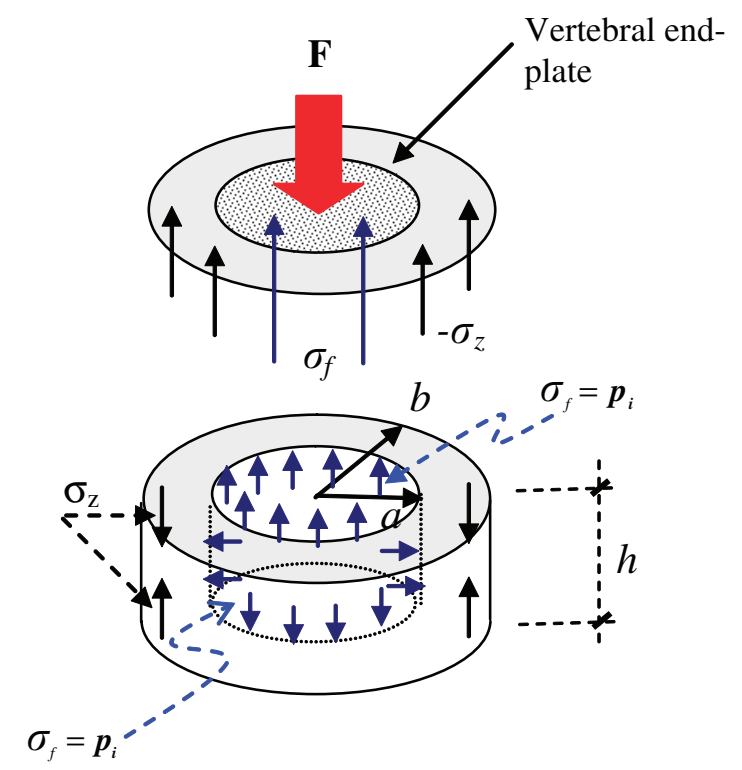

Figure 4: Normal stresses $\sigma_{f}$ and $\sigma_{z}$ under the applied compressive force $F$.

Then, substuting for $u_{a}$ from eqn (16) into eqn $12 \mathrm{c}$, we obtain

$$
p_{i}=-\frac{1-2 v}{2}\left(\frac{b^{2}-a^{2}}{a^{2}(1-2 v)+b^{2}(1+v)}\right) \sigma_{z} .
$$

The axial stress in the annulus is obtained by substituting the expression for $p_{i}$ from eqn (17) into eqn (14) as

$$
\sigma_{z}=-\frac{2}{\pi}\left(\frac{F}{b^{2}-a^{2}}\right)\left(\frac{a^{2}(1-2 v)+b^{2}(1+v)}{2 b^{2}(1+v)+a^{2}(3-6 v)}\right) .
$$

Then, from eqns (17) and (18), the NP pressure is expressed in terms of the applied compressive force $F$ as

$$
p_{i}=\frac{1-2 v}{\pi}\left(\frac{F}{3 a^{2}(1-2 v)+2 b^{2}(1+v)}\right) .
$$

From eqns (18) and (12c), by equating the expressions for $F$, we get the radial deformation at the inner surface as

$$
u_{a}=\frac{1}{\pi} \frac{1}{E}\left(\frac{F}{b^{2}-a^{2}}\right)\left(\frac{a^{3}(1-2 v)+a b^{2}}{2 b^{2}(1+v)+a^{2}(3-6 v)}\right) .
$$

From eqns (20) and (9), we get the axial deformation as

$$
w_{h}=\frac{2}{\pi} \frac{1}{E}\left(\frac{F}{b^{2}-a^{2}}\right)\left(\frac{h\left(a^{2}(1-2 v)+b^{2}\right)}{2 b^{2}(1+v)+a^{2}(3-6 v)}\right) .
$$


By substituting eqn (20) into eqn (13b), the expression for $u_{b}$ (the radial deformation at the outer surface of the annulus) is obtained as

$$
u_{b}=\frac{2}{\pi} \frac{1}{E}\left(\frac{F}{b^{2}-a^{2}}\right)\left(\frac{a^{2} b(1-2 v)+b^{3}}{2 b^{2}(1+v)+a^{2}(3-6 v)}\right) .
$$

Finally, from eqns (12a, b) and (20), we obtain the expressions for $\sigma_{r}$ and $\sigma_{\theta}$ in terms of applied load $F$ as

$$
\begin{aligned}
& \sigma_{r}=\frac{1-2 v}{\pi}\left(\frac{F}{b^{2}-a^{2}}\right)\left(\frac{a^{2}}{2 b^{2}(1+v)+a^{2}(3-6 v)}\right)\left(1-\frac{b^{2}}{r^{2}}\right), \\
& \sigma_{\theta}=\frac{1-2 v}{\pi}\left(\frac{F}{b^{2}-a^{2}}\right)\left(\frac{a^{2}}{2 b^{2}(1+v)+a^{2}(3-6 v)}\right)\left(1+\frac{b^{2}}{r^{2}}\right) .
\end{aligned}
$$

It is seen that as the disc gets loaded in compression (by the force $F$ ), (1) both $\sigma_{z}$ and $p_{i}$ increase, by virtue of eqns (18) and (19); (2) the increased $p_{i}$ (which is a function of $F$, as per eqn (19)) causes $\sigma_{r}$, $\sigma_{\theta}$ and $u_{b}$ to increase by virtue of eqns (22)-(24); (3) the axial (shortening) deformation $w_{h}$ increases by the virtue of eqn (21). Finally, the stresses $\left(\sigma_{r}, \sigma_{\theta}\right.$ and $\left.\sigma_{z}\right)$ are expressed in terms of $F$ by eqns (18), (23) and (24), while the deformations $\left(u_{\mathrm{a}}, u_{\mathrm{b}}\right.$ and $\left.w_{h}\right)$ are expressed in terms of $F$ by means of eqns (20)- (22).

\section{MECHANISM AND COMPUTATION OF DISC DEFORMATION}

The NP gets pressurized when the load $F$ acts on it, as per eqn (19). All the stresses increase with loading as per eqns (18), (23) and (24), and so does $E$ according to eqn (1). Now $E$ (the elastic modulus corresponding to the deformed state of the disc under load $F$ ) will be greater than its value in the unloaded state of the disc, as per eqn (1). Hence, as per eqns (21) and (22), both the axial and the radial deformations will be contained.

This is attributed to the disc design, wherein the annulus contains the NP. This dependency of $E$ on $p_{i}$, and hence on $F$ and the disc annulus stress state represented by $\sigma_{z}$ was also reported by Shirazi-Adl [14] and Ranu $[17,18]$, based on the experimental and finite element analysis of the annulus. The following procedure is followed to determine the disc deformation in response to compressive load.

Step 1

Starting from the unloaded state, $\sigma_{z 0}=0$, for which $E=E_{0}$ as per eqn (1).

1. Now an incremental compressive force of $\Delta F_{1}=1 \mathrm{~N}$ is applied on the unstressed disc of dimensions $\left(a_{0}, b_{0}\right.$ and $\left.h_{0}\right)$, and the incremental stresses $\left(\Delta \sigma_{r 1}, \Delta \sigma_{\theta 1}\right.$ and $\left.\Delta \sigma_{z 1}\right)$ are computed based on eqns (18), (23) and (24).

2. Next,the maximum value of these three stresses $\left(\Delta \sigma_{z 1}, \Delta \sigma_{r 1}\right.$ and $\left.\Delta \sigma_{\theta 1}\right)$, which happens to be $\Delta \sigma_{z 1}$, is noted. Then, based on $\Delta \sigma_{Z 1}, E=E_{\mathrm{I}}$ is computed according to the relation (based on eqn (1)): $E_{1}=E_{0}(=4.2)+373.3\left\{\left|\Delta \sigma_{z 1}\right|\right\}^{0.473}$.

3. The disc deformations $\left(w_{h 1}, u_{a 1}\right.$ and $\left.u_{b 1}\right)$, corresponding to the incremental stresses, are also computed from eqns (20)- (22), based on the above calculated value of $E=E_{1}$.

4. The disc geometry is now updated to $h_{1}=h_{0}-w_{h 1}, a_{1}=a_{0}+u_{a 1}, b_{1}=b_{0}+u_{b 1}$.

Step 2

1. Again, an incremental $\Delta F_{2}=1 \mathrm{~N}$ is applied on the deformed geometry of the disc $\left(a_{1}, b_{1}\right.$ and $\left.h_{1}\right)$, and incremental stresses $\left(\Delta \sigma_{r 2}, \Delta \sigma_{\theta 2}\right.$ and $\left.\Delta \sigma_{z 2}\right)$ are evaluated.

2. Next, the maximum value of these three stresses $\left(\Delta \sigma_{z 2}, \Delta \sigma_{r 2}\right.$ and $\left.\Delta \sigma_{\theta 2}\right)$ is noted, which happens to be $\Delta \sigma_{Z 2}$. 
3. The stress state is upgraded by adding $\Delta \sigma_{z 2}$ to $\Delta \sigma_{z 1}$, and $E_{2}$ is computed based on eqn (1) as: $E_{2}=E_{0}(=4.2)+373.3\left\{\left|\left[\Delta \sigma_{z 1}+\Delta \sigma_{z 2}\right]\right|\right\}^{0.473}$.

4. Then the incremental disc deformations $\left(w_{h 2}, u_{a 2}\right.$ and $\left.u_{b 2}\right)$ are determined corresponding to $\left(\Delta \sigma_{z 2}\right.$, $\Delta \sigma_{r 2}$ and $\Delta \sigma_{\theta 2}$ ), with $E_{2}$ as the updated annulus modulus. The total disc deformation is now: $\mathrm{w}_{h 1}+w_{h 2}, u_{a 1}+u_{a 2}, u_{b 1}+u_{b 2}$.

5. The deformed disc geometry is now updated to: $h_{2}=h_{1}-\left(w_{h 1}+w_{h 2}\right), a_{2}=a_{1}+\left(u_{a 1}+u_{a 2}\right)$, $b_{2}=b_{1}+\left(u_{b 1}+u_{b 2}\right)$.

Step 3

Step 2 is repeated until the total compressive force reaches $2000 \mathrm{~N}$ in order to obtain the final deformed geometry at the desired applied load.

The resulting graphs of disc deformations $w_{h}, u_{a}$ and $u_{b}$ vs. force $(F)$ are depicted in Fig. 5. The deformed geometry of the disc for $F=500 \mathrm{~N}, 1000 \mathrm{~N}, 1500 \mathrm{~N}$ and $2000 \mathrm{~N}$ are shown in Fig. 6, so as to depict the 'disc-hardening' effect whereby the disc deformations do not increase linearly with $F$.

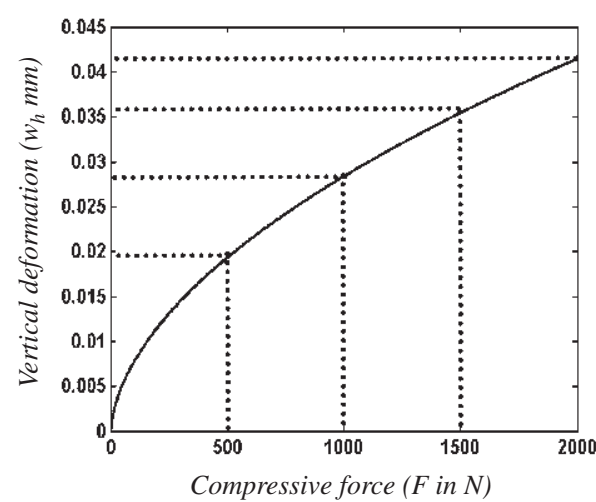

(a)

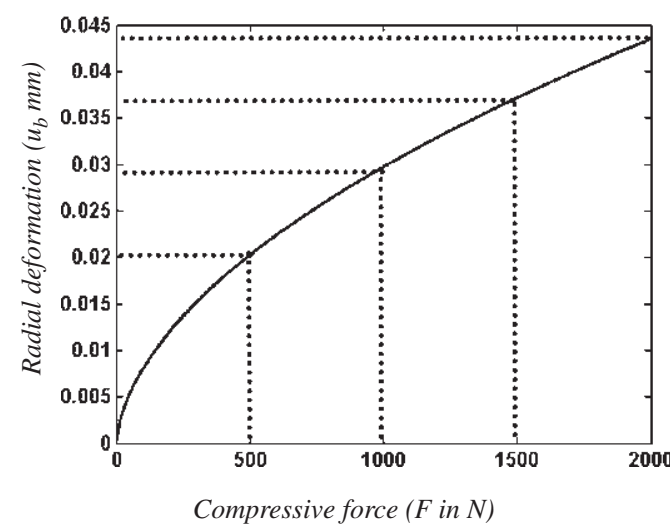

(c)

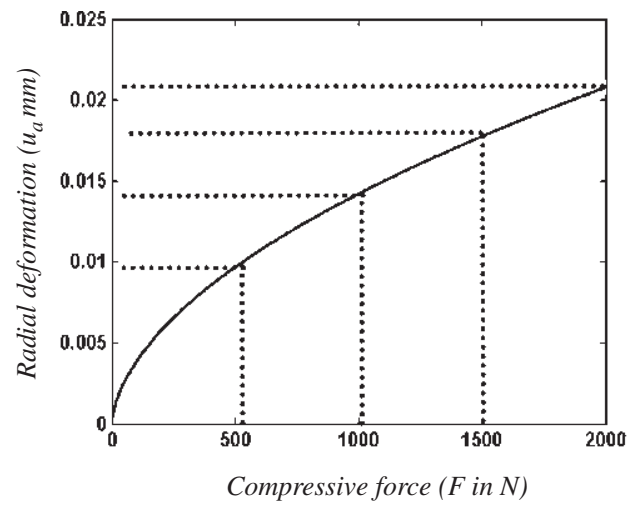

(b)

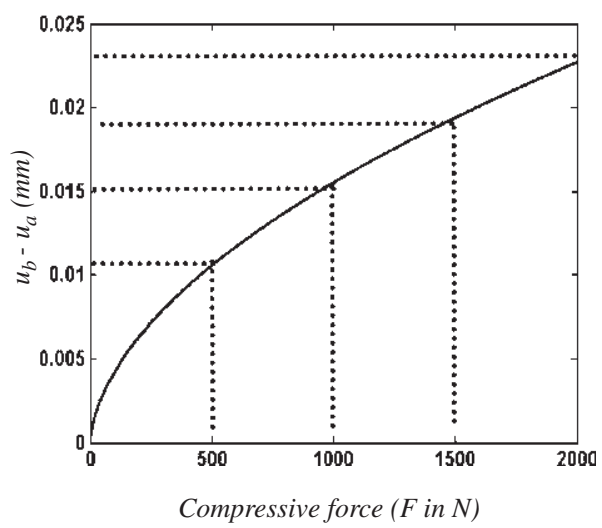

(d)

Figure 5: (a) Disc vertical deformation vs. compressive force on the annulus. (b) Radial bulge at $r=a$ vs. compressive force on the annulus. (c) Disc radial bulge at $r=b$ vs. compressive force. (d) Disc $u_{b}-u_{a}$ vs. $F$. The initial disc geometrical parameters adopted are $a=11 \mathrm{~mm}, b=25 \mathrm{~mm}$ and $h=11 \mathrm{~mm}$, and the annulus residual modulus $E_{0}$ is taken to be $4.2 \mathrm{MPa}$. 

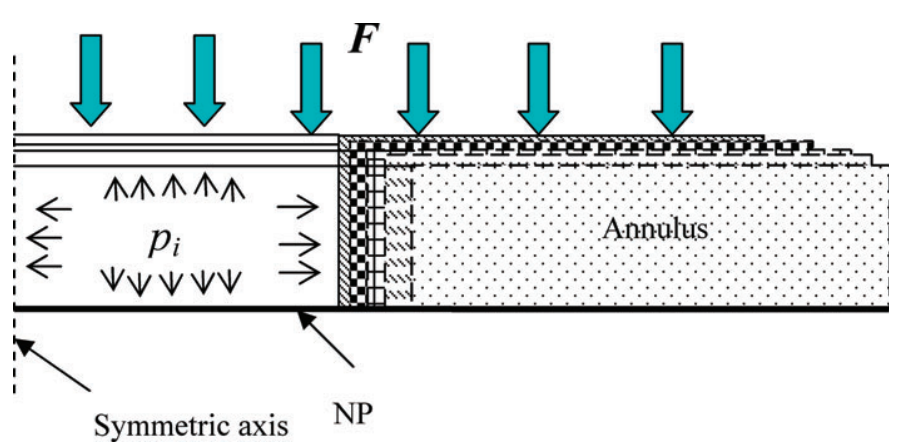

Figure 6: Graphical representation of disc deformation from the unloaded state until the compressive load of $2000 \mathrm{~N}$ is reached. represents the unloaded state; disc geometry parameters are $a=11 \mathrm{~mm}, b=25 \mathrm{~mm}, h=11 \mathrm{~mm}$. corresponds to the disc deformed state after $500 \mathrm{~N}$; the deformed disc geometry parameters are $a=11.009 \mathrm{~mm}, b=25.02 \mathrm{~mm}$, $h=10.99 \mathrm{~mm}$. EG corresponds to the deformed state after $1000 \mathrm{~N}$; disc geometry parameters are $a=11.014 \mathrm{~mm}, b=25.029 \mathrm{~mm}, h=10.975 \mathrm{~mm}$. the state after $1500 \mathrm{~N}$; disc geometry parameters are $a=11.018 \mathrm{~mm}, b=25.037 \mathrm{~mm}$, $h=10.965 \mathrm{~mm}$. $\mathrm{J}$ represents the final disc deformed state when loaded by $2000 \mathrm{~N}$; the corresponding final disc geometry parameters are $a=11.02 \mathrm{~mm}, b=25.044 \mathrm{~mm}$, $h=10.96 \mathrm{~mm}$.

\section{DISC HERNIATION, BACK PAIN AND NUCLEOTOMY}

If the load $F$ becomes very large, $\sigma_{\theta}$ would exceed the sustainable value and cause the annulus to develop radial cracks. Then the NP breaks through the annulus. A herniated disc occurs most often in the lumbar region of the spine, especially at the L4-L5 ( $\mathrm{L}=$ lumbar). This is because the lumbar spine carries most of the body's weight. People between the ages of 30 and 50 years appear to be more vulnerable because the elasticity and water content of the NP decreases with age. The pain resulting from herniation may be combined with radiculopathy (neurological deficit). The deficit may include numbness, weakness and reflex loss. These changes are caused by nerve compression, created by pressure from interior disc material. Percutaneous nucleotomy is carried out in order to remove the NP from the sequestered disc and thereby alleviate the back pain [19]. A probe is inserted into the centre of the herniated disc under fluoroscope monitoring and the NP is removed through the probe. The analysis for (1) volume aspiration of the NP fluid with respect to the time for different external suction pressures and (2) the pressure drop in the NP fluid with respect to the time was reported by Ghista et al. [13].

\section{THE NUCLEOTOMIZED DISC: GEOMETRY, STRESSES AND DISPLACEMENTS}

For the nucleotomized disc, only the axial equilibrium needs to be satisfied as there is no internal pressure, as given in eqn (3). Note that the radial and circumferential hoop stresses are identically equal to zero. Hence, the solution of eqn (3), with the boundary conditions $w=w_{h \text {,nu }}$ at $h=z$ and $w=0$ at $h=0$, is given by

$$
w=w_{\mathrm{nu}}=-w_{h, \mathrm{nu}} \frac{z}{h}
$$


The circumferential strain is related to the axial strain (by the Poisson's ratio) as

$$
\varepsilon_{\theta}=\frac{u_{\mathrm{nu}}}{r}=-v \varepsilon_{z}=-v \frac{\mathrm{d} w_{\mathrm{nu}}}{\mathrm{d} z}=v \frac{w_{h, \mathrm{nu}}}{h} .
$$

Hence, the radial displacements at $r=a$ and $r=b$ for the nucleotomized disc are given by

$$
u_{a, \mathrm{nu}}=v \frac{w_{h, \mathrm{nu}}}{h} a \quad \text { and } \quad u_{b, \mathrm{nu}}=v \frac{w_{h, \mathrm{nu}}}{h} b .
$$

\subsection{Stress analysis for a vertical loading on the nucleotomised disc}

For a vertically applied force $F$, the equilibrium of the disc is shown in Fig. 7; the minus sign is employed because the axial stress $\sigma_{z, \text { nu }}$ (assumed to be tensile) acts on the vertebral end-plate and the axial stress $\sigma_{z, \text { nu }}$ in the annulus is hence given by

$$
\sigma_{z, \mathrm{nu}}=-\frac{1}{\pi}\left(\frac{F}{b^{2}-a^{2}}\right) .
$$

Using Hooke's law, the axial deformation is related to $\sigma_{z, \text { nu }}$ and hence to the applied force $F$, so that the decrease in disc height

$$
w_{h, \mathrm{nu}}=\frac{1}{\pi} \frac{1}{E}\left(\frac{F h}{b^{2}-a^{2}}\right) .
$$

Then, from eqns (27) and (29), the radial expansion of the disc at $r=a$,

$$
u_{a, \mathrm{nu}}=\frac{1}{\pi} \frac{v}{E}\left(\frac{F a}{b^{2}-a^{2}}\right) .
$$
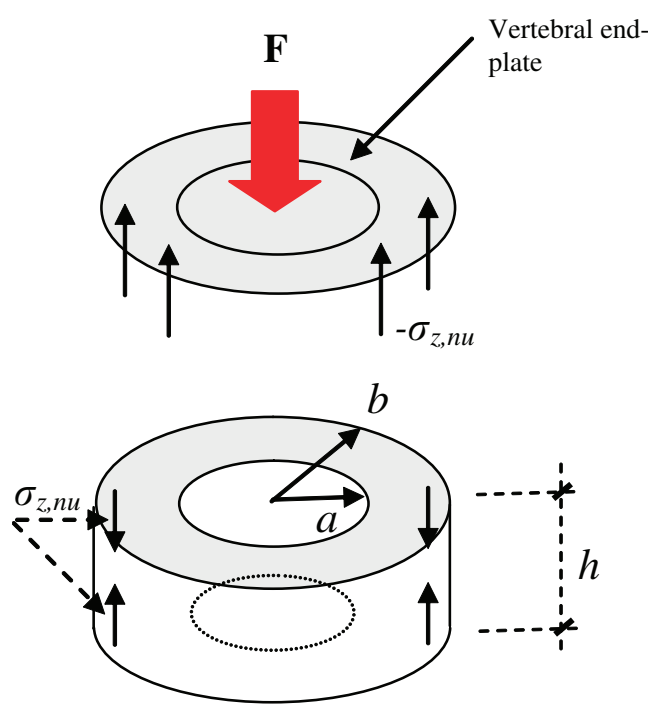

Figure 7: Normal stress $\sigma_{z, \text { nu }}$ equilibrating the applied force $F$ in a nucleotomized disc. 
Similarly, the radial expansion of the disc at $r=b$ is given by

$$
u_{b, \mathrm{nu}}=\frac{1}{\pi} \frac{v}{E}\left(\frac{F b}{b^{2}-a^{2}}\right) \quad \text { or } \quad u_{b, \mathrm{nu}}=\frac{b u_{a, \mathrm{nu}}}{a} .
$$

7 MECHANISM OF DISC DEFORMATION IN THE NUCLEOTOMIZED DISC

The same procedure as outlined in Section 4 is used to determine the incremental and final deformations of the nucleotomized disc under a uniaxial compressive load of $2000 \mathrm{~N}$. The resulting graphs of disc deformations $w_{h, \text { nu }}, u_{a, \text { nu }}, u_{b, \text { nu }}$ vs. $F$ and $\left(u_{b, \text { nu }}-u_{a, \text { nu }}\right)$ vs. $F$ are plotted in Fig. 8 alongside the deformations of the normal disc in order to provide a comparison. Also shown in Fig. 9 are the deformed disc geometries for $F=0, F=500 \mathrm{~N}, F=1000 \mathrm{~N}, F=1500 \mathrm{~N}$ and $F=2000 \mathrm{~N}$.

It is seen that the nucleotomized disc has considerably greater deformations than the normal disc. These deformations can result in compression of the nerve structures as well as the facet joints. Thus, the removal of the NP has adverse effects like disc collapse and excessive radial bulging. This trend is experimentally demonstrated by Meakin et al. [20] and Judith et al. [21].

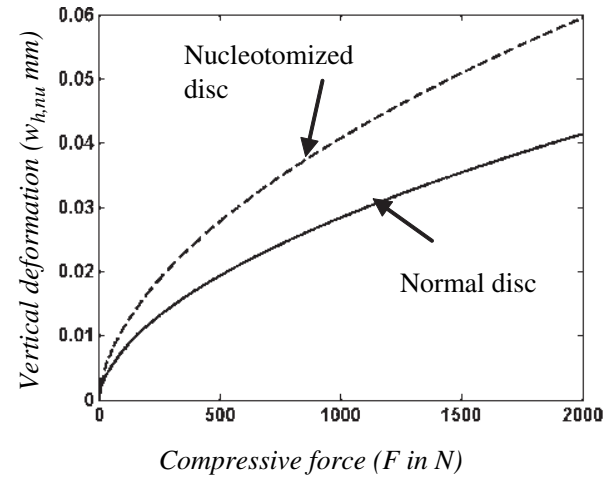

(a)

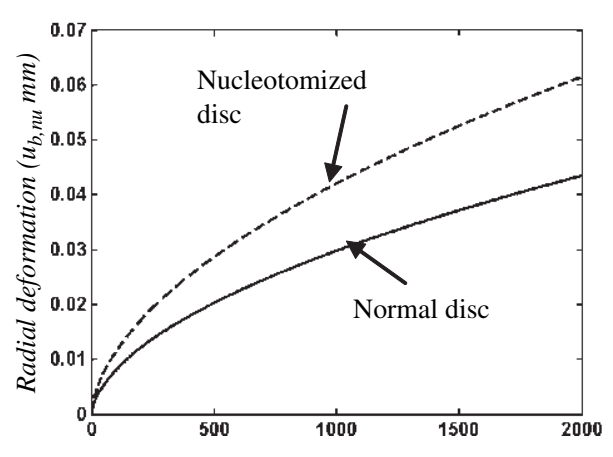

Compressive force ( $F$ in $N$ )

(c)

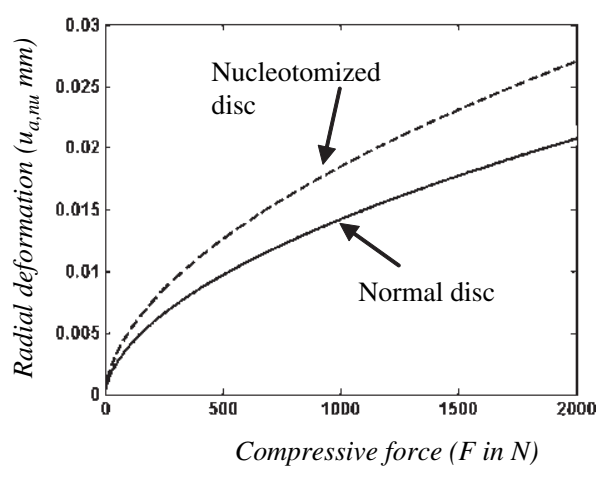

(b)

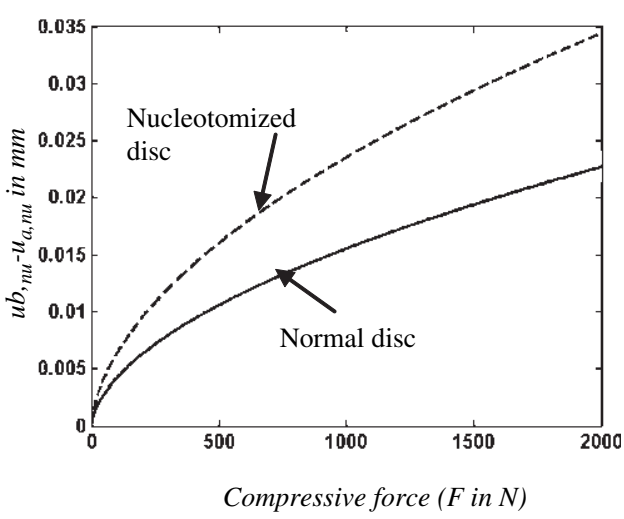

(d)

Figure 8: (a) Disc vertical deformation vs. compressive force on the annulus with and without NP. (b) Disc $u_{a, \text { nu }}$ vs. $F$ with and without NP. (c) Disc $u_{b, \text { nu }}$ vs. $F$ with and without NP. (d) Disc $\left(u_{b, \mathrm{nu}}-u_{a, \mathrm{nu}}\right)$ vs. $F$ with and without NP. The initial disc geometric parameters adopted are $a=11 \mathrm{~mm}, b=25 \mathrm{~mm}$ and $h=11 \mathrm{~mm}$ and the residual modulus $E_{0}$ is $4.2 \mathrm{MPa}$. 


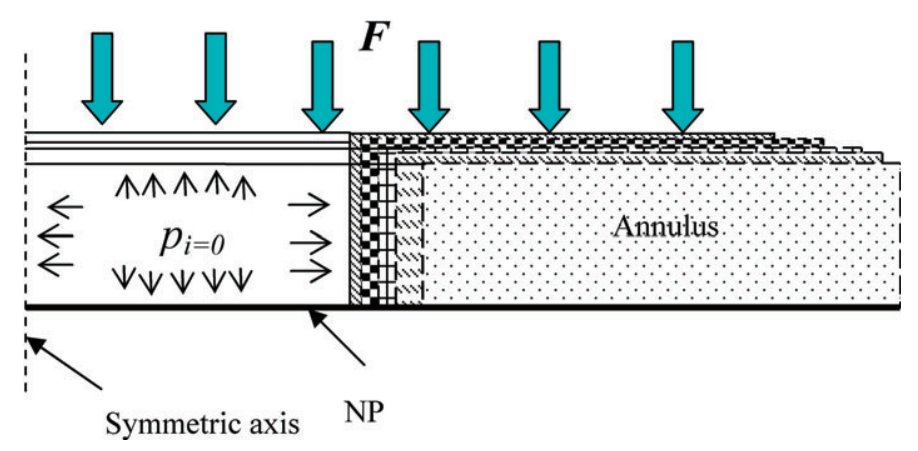

Figure 9: Graphical representation of the nucleotomized disc deformation from the unloaded state till the compressive load of $2000 \mathrm{~N}$. represents the unloaded state; disc geometry parameters are $a=11 \mathrm{~mm}, b=25 \mathrm{~mm}, h=11 \mathrm{~mm}$. 01 corresponds to the disc deformed state after $500 \mathrm{~N}$; geometry parameters are $a=11.014 \mathrm{~mm}, b=25.029 \mathrm{~mm}$, $h=10.972 \mathrm{~mm}$. EQj corresponds to the deformed state after $1000 \mathrm{~N}$; geometry parameters are $a=11.018 \mathrm{~mm}, b=25.041 \mathrm{~mm}, h=10.959 \mathrm{~mm}$. . $\mathrm{N}$; geometry parameters are $a=11.023 \mathrm{~mm}, b=25.051 \mathrm{~mm}, h=10.949 \mathrm{~mm}$. $\mathrm{J}$ represents the final disc deformed state when loaded by $2000 \mathrm{~N}$; the corresponding final disc geometry parameters are $a=11.026 \mathrm{~mm}, b=25.061 \mathrm{~mm}, h=10.94 \mathrm{~mm}$.

\section{CONCLUSION: THE IVD AS AN OPTIMAL STRUCTURE}

Based on these results, in order to retain the stress-stiffening characteristic of the disc and mimic the normal disc load-deformation behaviour, it is not advisable to carry out nucleotomy on herniated discs. Instead, it is advisable to replace the NP with a gel-filled balloon [22] in the case of disc herniation.

This paper clearly illustrates the natural anatomical-physiological design of the IVD as an optimal load-bearing and deformation-containing structure. This is because of the composite design of the IVD, in which the NP is enclosed by the annulus. Thus, when the IVD is loaded, the NP gets pressurized, its annulus stress increases, the annulus (stress-dependent) modulus increases and, hence, the annulus deformation is contained. This is the salient feature of the IVD as an optimal structure, namely its ability to contain its axial and radial deformations under increased loading.

$b \quad$ outer radius of the annulus

NOMENCLATURE

$a \quad$ inner radius of the annulus

$h \quad$ height of the annulus

$p_{i} \quad$ pressure of the NP

$F \quad$ compressive load

$\sigma_{f} \quad$ induced fluid pressure

$\sigma_{z} \quad$ compressive stress induced in the annulus

$\sigma_{\theta} \quad$ circumferential stress induced in the annulus

$\sigma_{r} \quad$ radial stress induced in the annulus,

$v \quad$ Poisson's ratio

E Young's modulus

$\varepsilon \quad$ strain 


\section{REFERENCES}

[1] Ghista, D.N., Fan, S.C., Ramakrishna, K. \& Sridhar, I., The human lumbar vertebral body as an intrinsic, functionally optimal structure. International Journal of Design and Nature, 1(1), pp. 34-47, 2007.

[2] Humzah, M.D. \& Soames, R.W., Human intervertebral disc: structure and function. The Anatomical Record, 220(4), pp. 337-356, 1988.

[3] http://www.spineuniverse.com/displayarticle.php/article1431.html (accessed on 10th September 2005)

[4] Shirazi-Adl, A., On the fibre composite material models of disc annulus-comparison of predicted stresses. Journal of Biomechanics, 22(4), pp. 357-365, 1989.

[5] Shirazi-Adl, A., Finite-element simulation of changes in the fluid content of human lumbar discs: mechanical and clinical implications. Spine, 17(2), pp. 206-212, 1990.

[6] Fagan, M.J., Julian, S., Siddall, D.J. \& Mohsen, A.M., Patient-specific spine models. Part 1: Finite element analysis of the lumbar intervertebral disc - a material sensitivity study. Proceedings of the Institution of Mechanical Engineers, Part H: Journal of Engineering in Medicine, 216(5), pp. 299-314, 2002.

[7] Lin, H.S., Liu, Y.K., Ray, G. \& Nikravesh, P., Systems identification for material properties of the intervertebral joint. Journal of Biomechanics, 11(2), pp. 1-14. 1978.

[8] Belytschko, T., Kulak, R.F., Schultz, A.B. \& Galante, J.O., Finite element stress analysis of an intervertebral disc. Journal of Biomechanics, 7(3), pp. 277-285, 1974.

[9] Wu, H.C. \& Yao, R.F., Mechanical behavior of the human annulus fibrosus. Journal of Biomechanics, 9(1), pp. 1-7, 1976.

[10] Kulak, R.F., Belytschko, T.B. \& Schultz, A.B., Nonlinear behavior of human intervertebral disc under axial load. Journal of Biomechanics, 9(6), pp. 377-386, 1976.

[11] Nick, D., Pope, M.H. \& Krag, M.H., Mechanical model for the human intervertebral disc. Journal of Biomechanics, 20(9), pp. 839-850, 1987.

[12] Martinez, J.B., Oloyede, V.O.A. \& Broom, N.D., Biomechanics of load-bearing of the intervertebral disc: an experimental and finite element model. Medical Engineering \& Physics, 19(2), pp. 145-156, 1997.

[13] Ghista, D.N., Subbaraj, S., Mazumdar, J. \& Rezaian, S.M., The biomechanics of back pain. IEEE Engineering in Medicine and Biology Magazine, 17(3), pp. 36-41, 1998.

[14] Shirazi-Adl, A., Srivastava, S.C. \& Ahmed, A.M., Stress analysis of the lumbar disc body unit in compression. Spine, 9, pp. 120-134, 1984.

[15] Yamada, H., Strength of Biological Materials, Williams and Wilkins: Baltimore, MD, 1970.

[16] Fagan, M.J., Julian, S. \& Mohsen, A.M., Finite element analysis in spine research. Proceedings of the Institution of Mechanical Engineers, Part H: Journal of Engineering in Medicine, 216, pp. 281-298, 2002.

[17] Ranu, H.S., Pressure-volume studies in human lumbar spinal discs. Southern Biomedical Engineering Conference-Proceedings, p. 263, 1995.

[18] Ranu, H.S., Denton, R.A., \& King, A.I., Pressure distribution under an intervertebral disc - an experimental study. Journal of Biomechanics, 12(10), pp. 807-812, 1979.

[19] Amoretti, N., Huchot, F., Flory, P., Brunner, P., Chevallier, P. \& Bruneton, J.N., Percutaneous nucleotomy: preliminary communication on a decompressor probe in percutaneous discectomy. Ten case reports. Clinical Imaging, 29, pp. 98-101, 2005.

[20] Meakin, J.R. \& Hukins, D.W.L., Effect of removing the nucleus pulposus on the deformation of the annulus fibrosus during compression of the intervertebral disc. Journal of Biomechanics, 33(5), pp. 575-580, 2000. 
[21] Judith, R.M., Thomas, W.R. \& David, W.L.H., The effect of partial removal of the nucleus pulposus from the intervertebral disc on the response of the human annulus fibrosus to compression. Clinical Biomechanics, 16, pp.121-128, 2001.

[22] Goel, V., Dooris, A.P., Dennis, M. \& Rengachary, S., Biomechanics of artificial disc. Advances in Spinal Fusion Molecular Science, Biomechanics, and Clinical Management, eds. K. Lewandrowski, L.W. Donald, D.J. Trantolo, M.J. Yaszemski \& A.A. White, Marcel Dekker: New York, 2004. 